\title{
On Interval Soft Sets with Applications
}

\author{
Xiaohong Zhang \\ Department of Mathematics, College of Arts and Sciences, \\ Shanghai Maritime University, Shanghai 201306, P.R. China \\ E-mail:zxhonghz@263.netorzhangxh@shmtu.edu.cn
}

Received 18 November 2012

Accepted 3 April 2013

\begin{abstract}
Interval set theory and soft set theory are mathematical tools for dealing with uncertainty information. As a combination of interval set and soft set, recently, we introduced the new notion of interval soft sets. In this paper we further research interval soft sets and its application. We investigate the tabular representation of interval soft sets, introduce the new concepts of interval choice values, and apply the theory of interval soft sets to solve a decision making problem by using two methods. We discuss some operations of interval soft sets, and construct some lattice structures. Moreover, we introduce the notion of soft equality in interval soft set theory, establish quotient algebra by soft equality relation, and discuss the application of soft equality relations in preconditioning of decision making.
\end{abstract}

Keywords: Soft set; Interval soft set; Decision making; Lattice; Soft equality relation.

\section{Introduction}

In order to describe and deal uncertainties, many mathematical tools are developed, for example, probability theory, fuzzy set theory, rough set theory $12,13,14$, interval sets theory ${ }^{20,21}$ and soft set theory ${ }^{10}$. This paper mainly related to the soft sets and interval sets.

Molodtsov ${ }^{10}$ introduced the concept of soft sets, which can be seen as a new mathematical tool for dealing with uncertainties. This so-called soft set theory is free from the difficulties affecting existing methods. Now, works on soft set theory are progressing rapidly. Maji, Ali and Sezgin et al. 1,8,18 defined some operations on soft sets and made some theoretical studies on the theory of soft sets. Jun et al. ${ }^{5,6}$ introduced the notion of soft BCK/BCIalgebras and discussed the applications of soft sets in ideal theory of BCK/BCI-algebras. Feng et al. ${ }^{3,4}$ applied soft set theory to the study of semirings and introduced the notions of rough soft sets and soft rough sets. Qin et al. ${ }^{16}$ introduced the notion of soft equality and established lattice structures and soft quotient algebras of soft sets. For the applications of soft set theory, Maji et al. ${ }^{9}$ introduced the notion of reduct-soft-set and described the application of soft set theory to a decision making problem using rough sets; Pei and Miao ${ }^{15}$ discussed the relationship between soft sets and information systems; Chen et al. ${ }^{2}$ presented a new definition of soft set parametrization reduction, and compared this definition to the related concept of attributes reduction in rough set theory.

For representing qualitative information, Yao ${ }^{20}$ proposed the notion of interval set, and the relationship among interval sets, rough sets and fuzzy sets are investigated in $21,22,23,24$. As a generalization of interval set, Zhang et al. ${ }^{25}$ introduced lattice-valued interval set and discussed its algebra operations. It is 
worth to show that interval set is not interval-valued fuzzy set. An interval set is an interval in the power set lattice based on a universal set and is a family of subsets of the universal set. Interval-valued fuzzy set can be regarded as a special lattice-valued interval set. Interval sets provide a new means for representing partially known concepts or for approximating undefinable concepts or complex concepts.

Every mathematical tool always has its limitations, and the integration of a variety of tools often can obtain the desired result of unexpected, therefore, the new theories by integrating different uncertainty mathematical tools become a hot of academic research (for example, probabilistic soft sets ${ }^{29}$, fuzzy soft sets ${ }^{7,17,19}$ and rough soft sets ${ }^{4}$ ). recently, we introduced the new notion of interval soft set by combining interval set theory and soft set theory in ${ }^{27}$ (It is differing from interval-valued fuzzy soft set $\left.{ }^{19}\right)$.

This paper is further study interval soft set theory in detail. The paper is organized as follows: In Section 2, we recall some notions and properties of interval sets, soft sets and interval soft sets. In Section 3, we discuss tabular representation of interval soft sets, and apply the theory of interval soft sets to solve a decision making problem by two methods. In section 4, we investigate some operations of interval soft sets and construct some lattice structures. In section 5, we introduce the notion of soft equality in interval soft set theory and establish quotient algebra by soft equality relation. Moreover, we describe the role of soft equality relations in preconditioning of decision making.

\section{Preliminaries}

Let $U$ be the universe set and $E$ the set of all possible parameters under consideration with respect to $U$. Usually, parameters are attributes, characteristic$\mathrm{s}$, or properties of objects in $U$. Molodtsov defined the notion of a soft set in the following way:

Definition 2.1 ${ }^{10}$ A pair $(F, A)$ is called a soft set over $U$, where $A \subseteq E$ and $F$ is a mapping given by $F: A \rightarrow P(U)$.

In other words, a soft set over $U$ is a parameter- ized family of subsets of $U$. For $e \in A, F(e)$ may be considered as the set of $e$-approximate elements of the soft set $(F, A)$.

Example 2.2 ${ }^{10}$ Suppose that there are six houses in the universe $U$ given by $U=\left\{h_{1}, h_{2}, h_{3}, h_{4}, h_{5}, h_{6}\right\}$ and $E=\left\{e_{1}, e_{2}, e_{3}, e_{4}, e_{5}\right\}$ is the set of parameter$\mathrm{s}$. Where $e_{1}$ stands for the parameter 'expensive', $e_{2}$ stands for the parameter 'beautiful', $e_{3}$ stands for the parameter 'wooden', $e_{4}$ stands for the parameter 'cheap' and $e_{5}$ stands for the parameter 'in the green surroundings'.

In this case, to define a soft set means to point out expensive houses, beautiful houses, and so on. The soft set $(F, E)$ may describe the 'attractiveness of the houses' which Mr.X is going to buy. Suppose that $F\left(e_{1}\right)=\left\{h_{2}, h_{4}\right\}, F\left(e_{2}\right)=\left\{h_{1}, h_{3}\right\}$, $F\left(e_{3}\right)=\left\{h_{3}, h_{4}, h_{5}\right\}, F\left(e_{4}\right)=\left\{h_{1}, h_{3}, h_{5}\right\}, F\left(e_{5}\right)=$ $\left\{h_{1}\right\}$. Then the soft set $(F, E)$ is a parameterized family $\left\{F\left(e_{i}\right): 1 \leqslant i \leqslant 5\right\}$ of subsets of $U$ and give us a collection of approximate descriptions of an object. $F\left(e_{1}\right)=\left\{h_{2}, h_{4}\right\}$ means 'houses $h_{2}$ and $h_{4}$ ' are 'expensive'.

In 1993, Yao ${ }^{20}$ introduced the notion of interval sets. Interval set is a new kind of sets, represented by a pair of sets, namely, its lower and upper bounds. Interval sets are interpreted in a similar way that interval numbers are introduced in interval analysis 11 .

Definition $2.3^{20}$ Let $U$ be a finite set, called the universe or the reference set, and $2^{U}$ be its power set. A subset of $2^{U}$ of the form

$$
\mathscr{A}=\left[A_{l}, A_{u}\right]=\left\{A \in 2^{U} \mid A_{l} \subseteq A \subseteq A_{u}\right\} .
$$

is called an interval set, where it is assumed $A_{l} \subseteq A_{u}$. The set of all interval sets is denoted by $I\left(2^{U}\right)$.

Remark 1: (1) In fact, the condition 'finite set' in Definition 2.3 is not necessary, we assume that $U$ is a non-empty set in this paper. (2) Degenerate interval sets of the form $[A, A]$ are equivalent to ordinary sets. Thus, interval sets may be considered as an extension of elementary sets.

Definition 2.4 ${ }^{20,24}$ Let $\cap, \cup$ and ${ }^{c}$ be the usual set intersection, union and complement defined on $2^{U}$, respectively. We define the following operations on 
$I\left(2^{U}\right)$ : for arbitrary interval sets $\mathscr{A}=\left[A_{l}, A_{u}\right]$ and $\mathscr{B}=\left[B_{l}, B_{u}\right]$,

$$
\begin{gathered}
\mathscr{A} \sqcap \mathscr{B}=\{A \cap B \mid A \in \mathscr{A}, B \in \mathscr{B}\}, \\
\mathscr{A} \sqcup \mathscr{B}=\{A \cup B \mid A \in \mathscr{A}, B \in \mathscr{B}\}, \\
\mathscr{A} \backslash \mathscr{B}=\{A-B \mid A \in \mathscr{A}, B \in \mathscr{B}\}, \\
\neg \mathscr{A}=\neg\left[A_{l}, A_{u}\right]=\left[A_{u}^{c}, A_{l}^{c}\right] .
\end{gathered}
$$

In fact, $\sqcap, \sqcup, \backslash$ can be explicitly computed by using the following formulas:

$$
\begin{aligned}
& \mathscr{A} \sqcap \mathscr{B}=\left[A_{l} \cap B_{l}, A_{u} \cap B_{u}\right], \\
& \mathscr{A} \sqcup \mathscr{B}=\left[A_{l} \cup B_{l}, A_{u} \cup B_{u}\right], \\
& \mathscr{A} \backslash \mathscr{B}=\left[A_{l}-B_{u}, A_{u}-B_{l}\right] .
\end{aligned}
$$

Moreover, the order relation $\sqsubseteq$ (inclusion of interval sets) on $I\left(2^{U}\right)$ be defined by:

$$
\mathscr{A} \sqsubseteq \mathscr{B} \Longleftrightarrow A_{l} \subseteq B_{l} \text { and } A_{u} \subseteq B_{u} .
$$

Based on this definition, for two interval sets $\mathscr{A}$ and $\mathscr{B}$,

$\mathscr{A}=\mathscr{B}$ if and only if $\mathscr{A} \sqsubseteq \mathscr{B}$ and $\mathscr{B} \sqsubseteq \mathscr{A}$.

As a combination of interval set and soft set, recently, we introduced the new notion of interval soft sets 27 .

Definition 2.5 Let $U$ be an initial universe set, $E$ a set of parameters and $A \subseteq E$. If $F$ is a mapping of $A$ into the set of all interval sets over $U$, that is, $F: A \rightarrow I\left(2^{U}\right)$, then the pair $(F, A)$ is called an interval soft set over $U$.

Example 2.6 Let $U$ be the set of houses under consideration, suppose $U=\left\{h_{1}, h_{2}, h_{3}, h_{4}, h_{5}, h_{6}\right\} . E$ is the set of parameters, each parameters is a word or a sentence, suppose $E=\left\{e_{1}\right.$ (beautiful), $e_{2}$ (wooden), $e_{3}$ (cheap), $e_{4}$ (in the green surroundings), $e_{5}$ (in good repair) $\}$. Putting

$$
\begin{aligned}
& F\left(e_{1}\right)=\left[\left\{h_{2}\right\},\left\{h_{2}, h_{4}\right\}\right], \\
& F\left(e_{2}\right)=\left[\left\{h_{1}\right\},\left\{h_{1}, h_{3}\right\}\right], \\
& F\left(e_{3}\right)=\left[\left\{h_{3}, h_{4}\right\},\left\{h_{3}, h_{4}\right\}\right], \\
& F\left(e_{4}\right)=\left[\left\{h_{5}\right\},\left\{h_{1}, h_{3}, h_{5}\right\}\right], \\
& F\left(e_{5}\right)=\left[\left\{h_{4}\right\},\left\{h_{1}, h_{4}, h_{6}\right\}\right] .
\end{aligned}
$$

Then $(F, E)$ is an interval soft set over $U$.

Consider soft set $(F, E)$, for every parameters $e$, $F(e)$ is a subset of universal set $U$, it can be regarded as all of objects which have the feature ' $e$ '. For interval soft set $(F, E)$, corresponding to every parameters $e, F(e)$ is a subset of $I\left(2^{U}\right)$, that is, $F(e)$ is a interval set, its lower bound (subset of $U$ ) can be regarded as all of objects which assuredly have the feature ' $e$ ', its upper bound (subset of $U$ ) can be regarded as all of objects which relatively have the feature ' $e$ '. In example 2.6, $F\left(e_{1}\right)=\left[\left\{h_{2}\right\},\left\{h_{2}, h_{4}\right\}\right]$, we can regard that the house in $\left\{h_{2}\right\}$ is assuredly 'expensive' $\left(e_{1}\right)$ and the house in $\left\{h_{2}, h_{4}\right\}$ are relatively 'expensive' $\left(e_{1}\right)$. Obviously, compared to soft sets, interval soft sets have more flexibility in the expression of uncertainty.

Remark 2: By Remark 1 (2), degenerate interval sets of the form $[A, A]$ are equivalent to set $A$. Therefore, if $(F, A)$ is a soft set, for all $e \in A, F(e)$ is equivalent to $[F(e), F(e)]$, then $(F, A)$ is also a interval soft set. That is, interval soft set is regarded a generalization of soft set.

\section{Tabular representation of interval soft sets and its application}

Similar to soft sets, we can represent interval soft sets in a tabular form. For interval soft set $(F, E)$ in Example 2.6, its tabular form as shown in Table 1.

Table 1. I-type tabular representation of an interval soft set $(F, E)$

\begin{tabular}{cccccc}
\hline & $e_{1}$ & $e_{2}$ & $e_{3}$ & $e_{4}$ & $e_{5}$ \\
\hline$h_{1}$ & {$[0,0]$} & {$[1,1]$} & {$[0,0]$} & {$[0,1]$} & {$[0,1]$} \\
$h_{2}$ & {$[1,1]$} & {$[0,0]$} & {$[0,0]$} & {$[0,0]$} & {$[0,0]$} \\
$h_{3}$ & {$[0,0]$} & {$[0,1]$} & {$[1,1]$} & {$[0,1]$} & {$[0,0]$} \\
$h_{4}$ & {$[0,1]$} & {$[0,0]$} & {$[1,1]$} & {$[0,0]$} & {$[1,1]$} \\
$h_{5}$ & {$[0,0]$} & {$[0,0]$} & {$[0,0]$} & {$[1,1]$} & {$[0,0]$} \\
$h_{6}$ & {$[0,0]$} & {$[0,0]$} & {$[0,0]$} & {$[0,0]$} & {$[0,1]$} \\
\hline
\end{tabular}

In Table 1 (call it I-type tabular representation), we use an interval number $c_{i j}=\left[a_{i j}, b_{i j}\right]$ to denote an attribute, where $c_{i j}$ are the entries in Table 1. For $F\left(e_{j}\right)=\left[A_{l}^{(j)}, A_{u}^{(j)}\right]$, we define

$$
\begin{aligned}
& \text { if } h_{i} \in A_{l}^{(j)} \text { then } a_{i j}=1 \text {, otherwise } a_{i j}=0 ; \\
& \text { if } h_{i} \in A_{u}^{(j)} \text { then } b_{i j}=1 \text {, otherwise } b_{i j}=0 .
\end{aligned}
$$

In fact, it has only three attribute value: $[0,0],[1,1]$ and $[0,1]$ in I-type tabular representation. Therefore, we can use an simple form (call it II-type tabular representation) as shown in Table 2. 
Table 2. II-type tabular representation of an interval soft set $(F, E)$

\begin{tabular}{cccccc}
\hline & $e_{1}$ & $e_{2}$ & $e_{3}$ & $e_{4}$ & $e_{5}$ \\
\hline$h_{1}$ & 0 & 1 & 0 & $\mathrm{u}$ & $\mathrm{u}$ \\
$h_{2}$ & 1 & 0 & 0 & 0 & 0 \\
$h_{3}$ & 0 & $\mathrm{u}$ & 1 & $\mathrm{u}$ & 0 \\
$h_{4}$ & $\mathrm{u}$ & 0 & 1 & 0 & 1 \\
$h_{5}$ & 0 & 0 & 0 & 1 & 0 \\
$h_{6}$ & 0 & 0 & 0 & 0 & $\mathrm{u}$ \\
\hline
\end{tabular}

In the above table, 0 stands for $[0,0], 1$ stands for $[1,1]$, "u" stands for $[0,1]$. Obviously, an interval soft set indeed is a simple information system (or information table, see ${ }^{12,13,14}$ ) in which the attributes take three values 0,1 and "u", it is differing from soft set whose tabular representation has only two attribute values.

In order to discuss the application of interval soft sets, we define the notion of interval choice value. For an object $h_{i} \in U$, its interval choice value is a interval number $v_{i}$ given by

$$
v_{i}=\left[v_{i}^{(1)}, v_{i}^{(2)}\right], v_{i}^{(1)}=\sum_{j} a_{i j}, v_{i}^{(2)}=\sum_{j} b_{i j} .
$$

where $a_{i j}, b_{i j}$ are defined in I-type tabular representation. For Table 1, the interval choice values are shown in Table 3.

Table 3. An interval soft set $(F, A)$ with interval choice values $(v)$

\begin{tabular}{ccccccc}
\hline & $e_{1}$ & $e_{2}$ & $e_{3}$ & $e_{4}$ & $e_{5}$ & $v$ \\
\hline$h_{1}$ & {$[0,0]$} & {$[1,1]$} & {$[0,0]$} & {$[0,1]$} & {$[0,1]$} & {$[1,3]$} \\
$h_{2}$ & {$[1,1]$} & {$[0,0]$} & {$[0,0]$} & {$[0,0]$} & {$[0,0]$} & {$[1,1]$} \\
$h_{3}$ & {$[0,0]$} & {$[0,1]$} & {$[1,1]$} & {$[0,1]$} & {$[0,0]$} & {$[1,3]$} \\
$h_{4}$ & {$[0,1]$} & {$[0,0]$} & {$[1,1]$} & {$[0,0]$} & {$[1,1]$} & {$[2,3]$} \\
$h_{5}$ & {$[0,0]$} & {$[0,0]$} & {$[0,0]$} & {$[1,1]$} & {$[0,0]$} & {$[1,1]$} \\
$h_{6}$ & {$[0,0]$} & {$[0,0]$} & {$[0,0]$} & {$[0,0]$} & {$[0,1]$} & {$[0,1]$} \\
\hline
\end{tabular}

Moreover, for II-type tabular representation of interval soft sets, we define interval choice value of object $h_{i}$ to be a formal interval number $v_{i}$ given by

$$
v_{i}=\left[v_{i}^{(1)}, v_{i}^{(2)}\right], v_{i}^{(1)}=\sum_{j, c_{i j}=1} c_{i j}, v_{i}^{(2)}=\sum_{j, c_{i j}=u} c_{i j} .
$$

where $c_{i j}$ are the entries in II-type tabular representation. For Table 2, the interval choice values are shown in Table 4.

Table 4. An interval soft set $(F, A)$ with interval choice values $(v)$

\begin{tabular}{ccccccc}
\hline & $e_{1}$ & $e_{2}$ & $e_{3}$ & $e_{4}$ & $e_{5}$ & $v$ \\
\hline$h_{1}$ & 0 & 1 & 0 & $\mathrm{u}$ & $\mathrm{u}$ & {$[1,2 \mathrm{u}]$} \\
$h_{2}$ & 1 & 0 & 0 & 0 & 0 & {$[1,0 \mathrm{u}]$} \\
$h_{3}$ & 0 & $\mathrm{u}$ & 1 & $\mathrm{u}$ & 0 & {$[1,2 \mathrm{u}]$} \\
$h_{4}$ & $\mathrm{u}$ & 0 & 1 & 0 & 1 & {$[2,1 \mathrm{u}]$} \\
$h_{5}$ & 0 & 0 & 0 & 1 & 0 & {$[1,0 \mathrm{u}]$} \\
$h_{6}$ & 0 & 0 & 0 & 0 & $\mathrm{u}$ & {$[0,1 \mathrm{u}]$} \\
\hline
\end{tabular}

Now, we consider the application of interval soft sets to decision making problems. Suppose that, Mr.X is interested to buy a house on the basis of his choice parameters 'beautiful' $\left(e_{1}\right)$, 'wooden' $\left(e_{2}\right)$, 'cheap' $\left(e_{3}\right)$, 'in the green surroundings' $\left(e_{4}\right)$, 'in good repair' $\left(e_{5}\right)$, which constitute the set $E=$ $\left\{e_{1}, e_{2}, e_{3}, e_{4}, e_{5}\right\}$. There are six houses under consideration, that is, $U=\left\{h_{1}, h_{2}, h_{3}, h_{4}, h_{5}, h_{6}\right\}$. Mr.X already give the evaluation data of six houses in Example 2.6 (interval soft set $(F, E)$ ). The problem is to select the house which is most suitable with the choice parameters of Mr.X (that is, the optimal house for Mr.X). By interval choice value (v) of interval soft set, we give the following algorithm:

1. input the house set $U$ and parameters set $E$ (may be a subset of $E$ );

2. input the interval soft set $(F, E)$;

3. give I-type tabular representation of interval soft set $(F, E)$;

4. compute interval choice value $v_{i}$;

5. find $k$ such that $v_{k}^{(1)}=\max v_{i}^{(1)}$ and $v_{k}^{(2)}=$ $\max _{\left\{m \mid v_{m}^{(1)}=\max v_{i}^{(1)}\right\}} v_{m}^{(2)}$.

Then $h_{k}$ is the choice object. If $k$ has more than one value, then any one of them could be chosen by Mr.X by using his option.

By the above algorithm, from Table 3, we get that the optimal house for Mr.X is $h_{4}$.

Similarly, for II-type tabular representation of interval soft set and interval choice value $v_{i}$, we can 
give a decision algorithm (it is omitted).

Moreover, we consider the problem using new method. In fact, interval choice value $v_{i}$ is a formal interval number. For convenience, we define a new choice value $\mu_{i}$ by putting "u" to a real number $\alpha \in[0,1]$ (it can be regarded as reliability level).

$\mu_{i}=\operatorname{Count}\left(\left\{j \mid c_{i j}=1\right\}\right)+\operatorname{Count}\left(\left\{j \mid c_{i j}=\mathrm{u}\right\}\right) \times \alpha$.

where $c_{i j}$ are the entries in II-type tabular representation, $\operatorname{Count}(A)$ denote the cardinal number of set $A$. For Table 2, the interval choice values are shown in Table 5 (putting $\alpha=0.7$ ).

Now, we give the following new algorithm:

1. input the house set $U$ and parameters set $E$ (may be a subset of $E$ );

2. input the interval soft set $(F, E)$;

3. give II-type tabular representation of interval soft set $(F, E)$;

4. choice a real number $\alpha \in[0,1]$, compute choice value $\mu_{i}$;

5. find $k$ such that $\mu_{k}=\max \mu_{i}$.

Then $h_{k}$ is the choice object. If $k$ has more than one value, then any one of them could be chosen by Mr.X by using his option.

By the above algorithm, from Table 5, we get that the optimal house for Mr.X is also $h_{4}$.

Remark 3: For interval soft sets, we can also discuss the parameters reduction which are different with attributes reduction in rough set theory $\left(\mathrm{see}^{2}\right)$. The aim of attributes reduction is to keep classification ability, however, the aim of parameters reduction is to keep the optimal objects. On parameters reduction of interval soft sets, we will discuss in the next paper.

\section{The lattice structures of interval soft sets}

Definition 4.1 ${ }^{27}$ The extended intersection of two interval soft sets $(F, A)$ and $(G, B)$ over a universe $U$ is the interval soft set $(H, C)=(F, A) \sqcap_{e}(G, B)$, where $C=A \cup B$, and for all $e \in C$,

$$
H(e)= \begin{cases}F(e) & \text { if } e \in A-B, \\ G(e) & \text { if } e \in B-A, \\ F(e) \sqcap G(e) & \text { if } e \in A \cap B .\end{cases}
$$

Definition 4.2 ${ }^{27}$ The extended union of two interval soft sets $(F, A)$ and $(G, B)$ over a universe $U$ is the interval soft set $(H, C)=(F, A) \sqcup_{e}(G, B)$, where $C=A \cup B$, and for all $e \in C$,

$$
H(e)= \begin{cases}F(e) & \text { if } e \in A-B, \\ G(e) & \text { if } e \in B-A, \\ F(e) \sqcup G(e) & \text { if } e \in A \cap B .\end{cases}
$$

Theorem $4.3^{27}$ Let $(F, A),(G, B)$ and $(H, C)$ be interval soft sets over the same universe $U$. Then

(1) $(F, A) \sqcap_{e}(F, A)=(F, A)$;

(2) $(F, A) \sqcap_{e}(G, B)=(G, B) \sqcap_{e}(F, A)$;

(3) $\left((F, A) \Pi_{e}(G, B)\right) \Pi_{e}(H, C)=(F, A) \Pi_{e}$ $\left((G, B) \sqcap_{e}(H, C)\right)$;

(4) $(F, A) \sqcup_{e}(F, A)=(F, A)$;

(5) $(F, A) \sqcup_{e}(G, B)=(G, B) \sqcup_{e}(F, A)$;

(6) $\left((F, A) \sqcup_{e}(G, B)\right) \sqcup_{e}(H, C)=(F, A) \sqcup_{e}$ $\left((G, B) \sqcup_{e}(H, C)\right)$.

Definition 4.4 $4^{27}$ The restricted intersection of two interval soft sets $(F, A)$ and $(G, B)$ over a universe $U$ is the interval soft set $(H, C)=(F, A) \sqcap_{r}(G, B)$, where $C=A \cap B$ and for all $e \in C, H(e)=F(e) \sqcap$ $G(e)$.

Definition 4.5 ${ }^{27}$ The restricted union of two interval soft sets $(F, A)$ and $(G, B)$ over a universe $U$ is the interval soft set $(H, C)=(F, A) \sqcup_{r}(G, B)$, where $C=A \cap B \neq \varnothing$ and for all $e \in C, H(e)=F(e) \sqcup G(e)$.

Theorem 4.6 ${ }^{27}$ Let $(F, A),(G, B)$ and $(H, C)$ be interval soft sets over the same universe $U$. Then

(1) $(F, A) \sqcap_{r}(F, A)=(F, A)$;

(2) $(F, A) \sqcap_{r}(G, B)=(G, B) \sqcap_{r}(F, A)$;

(3) $\left((F, A) \sqcap_{r}(G, B)\right) \sqcap_{r}(H, C)=(F, A) \sqcap_{r}$ $\left((G, B) \sqcap_{e}(H, C)\right)$; 
(4) $(F, A) \sqcup_{r}(F, A)=(F, A)$;

(5) $(F, A) \sqcup_{r}(G, B)=(G, B) \sqcup_{r}(F, A)$;

(6) $\left((F, A) \sqcup_{r}(G, B)\right) \sqcup_{r}(H, C)=(F, A) \sqcup_{r}$ $\left((G, B) \sqcup_{r}(H, C)\right)$.

For interval sets, operations $\sqcap$ and $\sqcup$ satisfy commutativity, associativity, idempotent and absorption $\left(\right.$ see $^{20,24}$ ). Using these properties, we can get following results.

Theorem 4.7 Let $(F, A)$ and $(G, B)$ be interval soft sets over the same universe $U$. Then

(1) $\left((F, A) \sqcup_{e}(G, B)\right) \sqcap_{r}(F, A)=(F, A)$;

(2) $\left((F, A) \sqcap_{r}(G, B)\right) \sqcup_{e}(F, A)=(F, A)$.

Proof. (1) Suppose that $(F, A) \sqcup_{e}(G, B)=(H, A \cup B)$ and $\left((F, A) \sqcup_{e}(G, B)\right) \sqcap_{r}(F, A)=(K,(A \cup B) \cap A)=$ $(K, A)$. For all $e \in A$,

(a) if $e \in B$, then $K(e)=H(e) \sqcap F(e)=(F(e) \sqcup$ $G(e)) \sqcap F(e)=F(e)$;

(b) if $e \notin B$, then $K(e)=H(e) \sqcap F(e)=F(e) \sqcap$ $F(e)=F(e)$;

Therefore, $\left((F, A) \sqcup_{e}(G, B)\right) \sqcap_{r}(F, A)=(F, A)$.

(2) Suppose that $(F, A) \sqcap_{r}(G, B)=(H, A \cap B)$ and $\left((F, A) \sqcap_{r}(G, B)\right) \sqcup_{e}(F, A)=(K,(A \cap B) \cup A)=$ $(K, A)$. For all $e \in A$,

(a) if $e \in B$, then $K(e)=H(e) \sqcup F(e)=(F(e) \sqcap$ $G(e)) \sqcup F(e)=F(e)$;

(b) if $e \notin B$, then $e \notin A \cap B$. By Definition 4.2, $K(e)=F(e)$.

Therefore, $\left((F, A) \sqcap_{r}(G, B)\right) \sqcup_{e}(F, A)=(F, A)$.

The above theorem shows that the absorption law with respect to operations $\sqcup_{e}$ and $\sqcap_{r}$ holds. Similarly, we can get that the absorption law with respect to operations $\sqcup_{r}$ and $\Pi_{e}$ holds as following theorem (the proof is omitted).

Theorem 4.8 Let $(F, A)$ and $(G, B)$ be interval soft sets over the same universe $U$. Then

(1) $\left((F, A) \sqcup_{r}(G, B)\right) \sqcap_{e}(F, A)=(F, A)$;

(2) $\left((F, A) \sqcap_{e}(G, B)\right) \sqcup_{r}(F, A)=(F, A)$.
In follows, the set of all interval soft sets over the universe $U$ and the parameter set $E$ is denote by $I S(U, E)$ :

$$
I S(U, E)=\left\{(F, A) \mid A \subseteq E, F: A \rightarrow I\left(2^{U}\right)\right\} .
$$

Theorem 4.9 Let $U$ be an universe set and $E$ a set of parameters. Then

(1) $\left(I S(U, E), \sqcup_{e}, \sqcap_{r}\right)$ is a distributive lattice.

(2) Denote by $\leqslant_{1}$ the order relation in lattice $\left(I S(U, E), \sqcup_{e}, \sqcap_{r}\right) . \quad$ For all $(F, A),(G, B) \in$ $I S(U, E), \quad(F, A) \leqslant 1 \quad(G, B) \Longleftrightarrow A \subseteq B$ and $F(e) \sqsubseteq G(e)$ for all $e \in E$.

Proof. (1) From Theorem 4.3, 4.6 and 4.7, we get that $\left(I S(U, E), \sqcup_{e}, \sqcap_{r}\right)$ is a lattice. We show that the following distributive law holds:

$(F, A) \sqcup_{e}\left((G, B) \sqcap_{r}(H, C)\right)=\left((F, A) \sqcup_{e}\right.$ $(G, B)) \sqcap_{r}\left((F, A) \sqcup_{e}(H, C)\right)$,

where $(F, A),(G, B),(H, C) \in I S(U, E)$. Suppose that

$(F, A) \sqcup_{e}\left((G, B) \sqcap_{r}(H, C)\right)=(K, A \cup(B \cap C))$,

$\left((F, A) \sqcup_{e}(G, B)\right) \sqcap_{r}\left((F, A) \sqcup_{e}(H, C)\right)=(L, A \cup$ $(B \cap C))$.

For any $e \in A \cup(B \cap C)$,

(a) if $e \notin A$, then $e \in B$ and $e \in C$. Thus, $K(e)=$ $G(e) \sqcap H(e)=L(e)$.

(b) if $e \in A, e \notin B, e \notin C$, then $K(e)=F(e)=$ $F(e) \sqcap F(e)=L(e)$.

(c) if $e \in A, e \in B, e \notin C$, then $K(e)=F(e)=$ $(F(e) \sqcup G(e)) \sqcap F(e)=L(e)$.

(d) if $e \in A, e \notin B, e \in C$, then $K(e)=F(e)=$ $F(e) \sqcap(F(e) \sqcup H(e))=L(e)$.

(e) if $e \in A, e \in B, e \in C$, then $K(e)=F(e) \sqcup$ $(G(e) \sqcap H(e))=(F(e) \sqcup G(e)) \sqcap(F(e) \sqcup H(e))=$ $L(e)$.

Therefore, the distributive law holds, this means that $\left(I S(U, E), \sqcup_{e}, \sqcap_{r}\right)$ is a distributive lattice.

(2) Assume that $(F, A) \leqslant_{1}(G, B)$. Then $(F, A) \sqcup_{e}$ $(G, B)=(G, B)$. By the definition of $\sqcup_{e}$, we have $A \cup B=B,(\forall e \in E) F(e) \sqcup G(e)=G(e)$. Hence, $A \subseteq B$ and $F(e) \sqsubseteq G(e)$ for all $e \in E$.

Conversely, assume that $A \subseteq B$ and $F(e) \sqsubseteq G(e)$ for all $e \in E$. By the definition of $\sqcup_{e}$, we get that $(F, A) \sqcup_{e}(G, B)=(G, B)$, it follows that $(F, A) \leqslant 1$ $(G, B)$. 
Theorem 4.10 Let $U$ be an universe set and $E$ a set of parameters. Then

$(F, A) \sqcup_{r}\left((G, B) \sqcap_{e}(H, C)\right)=\left((F, A) \sqcup_{r}\right.$ $(G, B)) \sqcap_{e}\left((F, A) \sqcup_{e}(H, C)\right)$,

where $(F, A),(G, B),(H, C) \in I S(U, E)$.

Proof. Suppose that

$$
\begin{aligned}
& (F, A) \sqcup_{r}\left((G, B) \sqcap_{e}(H, C)\right)=(K, A \cap(B \cup C)), \\
& \left((F, A) \sqcup_{r}(G, B)\right) \sqcap_{e}\left((F, A) \sqcup_{r}(H, C)\right)=(L, A \cap
\end{aligned}
$$

$(B \cup C))$.

For any $e \in A \cap(B \cup C)$, we have $e \in A$ and $e \in$ $(B \cup C)$. $L(e)$.

(a) if $e \notin B, e \notin C$, then $K(e)=F(e) \sqcup H(e)=$ $L(e)$.

(b) if $e \in B, e \notin C$, then $K(e)=F(e) \sqcup G(e)=$

(c) if $e \in B, e \in C$, then $K(e)=F(e) \sqcup(G(e) \sqcap$ $H(e))=(F(e) \sqcup G(e)) \sqcap(F(e) \sqcup H(e))=L(e)$.

Therefore, $\quad(F, A) \sqcup_{r}\left((G, B) \Pi_{e}(H, C)\right)=$ $\left((F, A) \sqcup_{r}(G, B)\right) \sqcap_{e}\left((F, A) \sqcup_{e}(H, C)\right)$.

By Theorem 4.3, 4.6, 4.8 and 4.10, we can get another lattice structure of interval soft sets.

Theorem 4.11 Let $U$ be an universe set and $E$ a set of parameters. Then

(1) $\left(I S(U, E), \sqcup_{r}, \sqcap_{e}\right)$ is a distributive lattice.

(2) Denote by $\leqslant_{2}$ the order relation in lattice $\left(I S(U, E), \sqcup_{r}, \sqcap_{e}\right) . \quad$ For all $(F, A),(G, B) \in$ $I S(U, E), \quad(F, A) \leqslant 2(G, B) \Longleftrightarrow B \subseteq A$ and $F(e) \sqsubseteq G(e)$ for all $e \in B$.

Proof. It is similar to Theorem 4.9.

\section{Soft equality relation and quotient algebras}

Definition 5.1 Let $(F, A),(G, B)$ be two interval soft sets over the universe $U$ and the parameter set $E$. $(F, A)$ is called I-type soft equal to $(G, B)$ denoted by $(F, A) \approx_{1}(G, B)$, if for all $e \in A \cup B$,

$e \in A \cap B$ implies $F(e)=G(e)$;

$e \in A-B$ implies $F(e)=[\varnothing, \varnothing]$;

$e \in B-A$ implies $G(e)=[\varnothing, \varnothing]$.
Example 5.2 Let $U=\left\{h_{1}, h_{2}, h_{3}, h_{4}, h_{5}, h_{6}\right\}$ and $E=$ $\left\{e_{1}, e_{2}, e_{3}, e_{4}, e_{5}\right\}$. Putting $A=\left\{e_{1}, e_{2}, e_{3}, e_{4}\right\}, B=$ $\left\{e_{1}, e_{2}, e_{3}\right\}, C=\left\{e_{1}, e_{2}, e_{3}, e_{5}\right\}$ and

$$
\begin{aligned}
& F\left(e_{1}\right)=G\left(e_{1}\right)=H\left(e_{1}\right)=\left[\left\{h_{2}\right\},\left\{h_{2}, h_{4}\right\}\right], \\
& F\left(e_{2}\right)=G\left(e_{2}\right)=H\left(e_{2}\right)=\left[\left\{h_{1}\right\},\left\{h_{1}, h_{3}\right\}\right], \\
& F\left(e_{3}\right)=G\left(e_{3}\right)=H\left(e_{3}\right)=\left[\left\{h_{3}, h_{4}\right\},\left\{h_{3}, h_{4}\right\}\right], \\
& F\left(e_{4}\right)=[\varnothing, \varnothing], \\
& H\left(e_{5}\right)=[U, U] .
\end{aligned}
$$

Then $(F, A) \approx_{1}(G, B)$. Obviously, $(F, A) \approx_{1}(H, C)$ and $(G, B) \approx_{1}(H, C)$ are not true.

Theorem $5.3 \approx_{1}$ is an equivalence relation on $I S(U, E)$.

Proof. It is easy to verify that $\approx_{1}$ is reflexive and symmetric.

Suppose that $(F, A) \approx_{1}(G, B)$ and $(G, B) \approx_{1}$ $(H, C)$. For all $e \in A \cup C$,

Case 1: $e \in A \cap C$. If $e \in B$, then $e \in A \cap B$ and $e \in B \cap C$, it follows that $F(e)=G(e)=H(e)$; if $e \notin B$, then $e \in A-B$ and $e \in C-B$, it follows that $F(e)=[\varnothing, \varnothing]=H(e)$.

Case 2: $e \in A-C$. It follows that $e \in A$ and $e \notin C$. If $e \in B$, then $e \in A \cap B$ and $e \in B-C$, so $F(e)=G(e)=[\varnothing, \varnothing]$; if $e \notin B$, then $e \in A-B$, so $F(e)=[\varnothing, \varnothing]$.

Case 3: $e \in C-A$. It follows that $e \in C$ and $e \notin A$. If $e \in B$, then $e \in B \cap C$ and $e \in B-A$, so $H(e)=G(e)=[\varnothing, \varnothing]$; if $e \notin B$, then $e \in C-B$, so $H(e)=[\varnothing, \varnothing]$.

This means that $\approx_{1}$ is transitive. Therefore, $\approx_{1}$ is an equivalence relation on $I S(U, E)$.

Theorem $5.4 \approx_{1}$ is a congruence relation with respect to operations $\sqcup_{e}$ and $\sqcap_{r}$. That is, if $(F, A) \approx_{1}$ $(G, B)$ and $(H, C) \approx_{1}(L, D)$, then

(1) $(F, A) \sqcup_{e}(H, C) \approx_{1}(G, B) \sqcup_{e}(L, D)$.

(2) $(F, A) \sqcap_{r}(H, C) \approx_{1}(G, B) \sqcap_{r}(L, D)$.

Proof. (1) Suppose that $(F, A) \sqcup_{e}(H, C)=(M, A \cup$ $C),(G, B) \sqcup_{e}(L, D)=(N, B \cup D)$. For all $e \in(A \cup$ $C) \cup(B \cup D)$,

Case 1: $e \in(A \cup C) \cap(B \cup D)$. That is, $e \in(A \cup C)$ and $e \in(B \cup D)$. Without loss of generality, we suppose that $e \in A$ and $e \in D$. 
If $e \in B$ and $e \in C$, then $e \in A \cap B$ and $e \in C \cap D$. It follows that $F(e)=G(e), H(e)=L(e)$, thus $M(e)=$ $F(e) \sqcup H(e)=G(e) \sqcup L(e)=N(e)$.

If $e \notin B$ and $e \in C$, then $e \in A-B$ and $e \in C \cap D$. It follows that $F(e)=[\varnothing, \varnothing], H(e)=L(e)$, thus $M(e)=F(e) \sqcup H(e)=H(e)=L(e)=N(e)$.

If $e \in B$ and $e \notin C$, then $e \in A \cap B$ and $e \in$ $D-C$. It follows that $F(e)=G(e), L(e)=[\varnothing, \varnothing]$, thus $M(e)=F(e)=G(e)=G(e) \sqcup L(e)=N(e)$.

If $e \notin B$ and $e \notin C$, then $e \in A-B$ and $e \in D-C$. It follows that $F(e)=[\varnothing, \varnothing], L(e)=[\varnothing, \varnothing]$, thus $M(e)=F(e)=[\varnothing, \varnothing]=L(e)=N(e)$.

Case 2: $e \in(A \cup C)-(B \cup D)$. That is, $e \in$ $(A \cup C), e \notin B$ and $e \notin D$.

If $e \in A$ and $e \in C$, then $e \in A-B$ and $e \in C-D$. It follows that $F(e)=H(e)=[\varnothing, \varnothing]$, thus $M(e)=$ $F(e) \sqcup H(e)=[\varnothing, \varnothing]$.

If $e \in A$ and $e \notin C$, then $e \in A-B$. It follows that $F(e)=[\varnothing, \varnothing]$, thus $M(e)=F(e)=[\varnothing, \varnothing]$.

If $e \notin A$ and $e \in C$, then $e \in C-D$. It follows that $H(e)=[\varnothing, \varnothing]$, thus $M(e)=H(e)=[\varnothing, \varnothing]$.

Case 3: $e \in(B \cup D)-(A \cup C)$. We can get $N(e)=[\varnothing, \varnothing]$ (the proof is similar to Case 2).

Therefore, $(F, A) \sqcup_{e}(H, C) \approx_{1}(G, B) \sqcup_{e}(L, D)$.

(2) Suppose that $(F, A) \sqcap_{r}(H, C)=(S, A \cap$ $C),(G, B) \sqcap_{r}(L, D)=(T, B \cap D)$. For all $e \in(A \cap$ $C) \cup(B \cap D)$,

Case 1: $e \in(A \cap C) \cap(B \cap D)$. That is, $e \in(A \cap B)$ and $e \in C \cap D$. It follows that $F(e)=G(e), H(e)=$ $L(e)$, thus $S(e)=F(e) \sqcap H(e)=G(e) \sqcap L(e)=T(e)$.

Case 2: $e \in(A \cap C)-(B \cap D)$. That is, $e \in A, e \in$ $C$ and $e \notin(B \cap D)$. It follows that $e \notin B$ or $e \notin D)$.

If $e \notin B$, then $e \in A-B$ and hence $F(e)=[\varnothing, \varnothing]$. It follows that $S(e)=F(e) \sqcap H(e)=[\varnothing, \varnothing]$.

If $e \notin D$, then $e \in C-D$ and hence $H(e)=[\varnothing, \varnothing]$. It follows that $S(e)=F(e) \sqcap H(e)=[\varnothing, \varnothing]$.

Case 3: $e \in(B \cap D)-(A \cap C)$. We can get $T(e)=[\varnothing, \varnothing]$ (the proof is similar to Case 2).

Therefore, $(F, A) \sqcap_{r}(H, C) \approx_{1}(G, B) \sqcap_{r}(L, D)$.

Let $(F, A) \approx_{1}=\left\{(G, B) \mid(G, B) \approx_{1}(F, A)\right\}$ be the congruence class and $I S(U, E) / \approx_{1}=\left\{(F, A) \approx_{1} \mid\right.$ $(F, A) \in I S(U, E)\}$. We define operations $\mathbb{U}_{1}$ and $\cap_{1}$ on $I S(U, E) / \approx_{1}$ as follows:

$$
(F, A)_{\approx_{1}} \uplus_{1}(G, B)_{\approx_{1}}=\left((F, A) \sqcup_{e}(G, B)\right)_{\approx_{1}} .
$$

$$
(F, A) \approx_{\approx_{1}} \cap_{1}(G, B) \approx_{1}=\left((F, A) \sqcap_{r}(G, B)\right)_{\approx_{1}} .
$$

By Theorem 5.4 we know that these operations are well defined. We call $\left(I S(U, E) / \approx_{1}, \mathbb{U}_{1}\right.$, П 1 1) the soft quotient algebra with respect to $\approx_{1}$ over the universe $U$ and the parameter set $E$. By Theorem 4.9 and applying the related results in universal algebra (see ${ }^{26}$ ), we can get following theorem (the proof is omitted).

Theorem 5.5 The soft quotient algebra $\left(I S(U, E) / \approx_{1}, \mathbb{U}_{1}, \cap_{1}\right)$ is a distributive lattice.

The above soft equality relation and soft quotient algebra are based on the operations $\sqcup_{e}$ and $\sqcap_{r}$. Similarly, we can establish another quotient algebra structures based on the operations $\sqcup_{r}$ and $\sqcap_{e}$. We list related concepts and results in the end of this section and omit the proofs.

Definition 5.6 Let $(F, A),(G, B)$ be two interval soft sets over the universe $U$ and the parameter set $E$. $(F, A)$ is called II-type soft equal to $(G, B)$ denoted by $(F, A) \approx_{2}(G, B)$, if for all $e \in A \cup B$,

$e \in A \cap B$ implies $F(e)=G(e)$;

$e \in A-B$ implies $F(e)=[U, U]$;

$e \in B-A$ implies $G(e)=[U, U]$.

For Example 5.2, we have $(G, B) \approx_{2}(H, C)$.

Theorem $5.7 \approx_{2}$ is an equivalence relation on $I S(U, E)$.

Theorem $5.8 \approx_{2}$ is a congruence relation with respect to operations $\sqcup_{r}$ and $\Pi_{e}$. That is, if $(F, A) \approx_{2}$ $(G, B)$ and $(H, C) \approx_{2}(L, D)$, then

(1) $(F, A) \sqcup_{r}(H, C) \approx_{2}(G, B) \sqcup_{r}(L, D)$.

(2) $(F, A) \sqcap_{e}(H, C) \approx_{2}(G, B) \sqcap_{e}(L, D)$.

Let $(F, A) \approx_{2}=\left\{(G, B) \mid(G, B) \approx_{2}(F, A)\right\}$ be the congruence class and $I S(U, E) / \approx_{2}=\left\{(F, A) \approx_{2} \mid\right.$ $(F, A) \in I S(U, E)\}$. We define operations $\uplus_{2}$ and $\mathrm{n}_{2}$ on $I S(U, E) / \approx_{2}$ as follows:

$$
\begin{aligned}
& (F, A)_{\approx_{2}} \uplus_{2}(G, B)_{\approx_{2}}=\left((F, A) \sqcup_{r}(G, B)\right)_{\approx_{2}} . \\
& (F, A)_{\approx_{2} \cap_{2}}(G, B)_{\approx_{2}}=\left((F, A) \sqcap_{e}(G, B)\right)_{\approx_{2}} .
\end{aligned}
$$


We call $\left(I S(U, E) / \approx_{2}, \mathbb{U}_{2}, \cap_{2}\right)$ the II-type soft quotient algebra with respect to $\approx_{2}$ over the universe $U$ and the parameter set $E$.

Theorem 5.9 The II-type soft quotient algebra $\left(I S(U, E) / \approx_{2}, \mathbb{U}_{2}, \cap_{2}\right)$ is a distributive lattice.

Finally, we discuss the application of soft equality relations to preconditioning of decision making.

Example 5.10 Let $U$ be the set of houses under consideration, suppose $U=\left\{h_{1}, h_{2}, h_{3}, h_{4}, h_{5}, h_{6}\right\} . E_{1}$ is the set of parameters, each parameters is a word or a sentence, suppose $E_{1}=\left\{e_{1}\right.$ (beautiful), $e_{2}$ (wooden), $e_{3}$ (cheap), $e_{4}$ (in the green surroundings), $e_{5}$ (in good repair), $e_{6}$ (expensive) $\}$. Putting

$$
\begin{aligned}
& G\left(e_{1}\right)=\left[\left\{h_{2}\right\},\left\{h_{2}, h_{4}\right\}\right], \\
& G\left(e_{2}\right)=\left[\left\{h_{1}\right\},\left\{h_{1}, h_{3}\right\}\right], \\
& G\left(e_{3}\right)=\left[\left\{h_{3}, h_{4}\right\},\left\{h_{3}, h_{4}\right\}\right], \\
& G\left(e_{4}\right)=\left[\left\{h_{5}\right\},\left\{h_{1}, h_{3}, h_{5}\right\}\right], \\
& G\left(e_{5}\right)=\left[\left\{h_{4}\right\},\left\{h_{1}, h_{4}, h_{6}\right\}\right], \\
& G\left(e_{6}\right)=[\varnothing, \varnothing] .
\end{aligned}
$$

Then $\left(G, E_{1}\right)$ is an interval soft set over $U$. Compared with interval soft set $(F, E)$ in Example 2.6, we know that $\left(G, E_{1}\right) \approx_{1}(F, E)$. Since $G\left(e_{6}\right)=[\varnothing, \varnothing]$, this means that all of houses under consideration are not expensive, therefore, $e_{6}$ is not considered in the decision problem to select houses.

By the above example, we give the following decision method by using soft set $\left(G, E_{1}\right)$ :

Step 1: from $\left(G, E_{1}\right)$, delete the parameters (attributes) whose valued are always $[0,0]$ in the I-type tabular representation of $\left(G, E_{1}\right)$, obtain a new interval soft set $(F, E)$ such that $\left(G, E_{1}\right) \approx_{1}(F, E)$ and $E \subseteq E_{1}$;

Step 2: for interval soft set $(F, E)$, apply the algorithms in Section 3.

This means that the soft equality relation $\approx_{1}$ is applied to preconditioning of decision making.

Similarly, the soft equality relation $\approx_{2}$ can be applied to preconditioning of decision making, therefore, the above decision method can be extended to following general method by using soft set $\left(H, E_{2}\right)$ :
Step 1: from $\left(H, E_{2}\right)$, delete the parameters (attributes) whose valued are always $[1,1]$ in the I-type tabular representation of $\left(H, E_{2}\right)$, obtain a new interval soft set $\left(G, E_{1}\right)$ such that $\left(H, E_{2}\right) \approx_{2}\left(G, E_{1}\right)$ and $E_{1} \subseteq E_{2}$;

Step 2: from $\left(G, E_{1}\right)$, delete the parameters (attributes) whose valued are always $[0,0]$ in the I-type tabular representation of $\left(G, E_{1}\right)$, obtain another new interval soft set $(F, E)$ such that $\left(G, E_{1}\right) \approx_{1}(F, E)$ and $E \subseteq E_{1}$;

Step 3: for interval soft set $(F, E)$, apply the algorithms in Section 3.

Example 5.11 Let $U$ be the set of houses under consideration, suppose $U=\left\{h_{1}, h_{2}, h_{3}, h_{4}, h_{5}, h_{6}\right\} . E_{2}$ is the set of parameters, each parameters is a word or a sentence, suppose $E_{2}=\left\{e_{1}\right.$ (beautiful), $e_{2}$ (wooden), $e_{3}$ (cheap), $e_{4}$ (in the green surroundings), $e_{5}$ (in good repair), $e_{6}$ (expensive), $e_{7}($ modern $\left.)\right\}$. Putting

$$
\begin{aligned}
& H\left(e_{1}\right)=\left[\left\{h_{2}\right\},\left\{h_{2}, h_{4}\right\}\right], \\
& H\left(e_{2}\right)=\left[\left\{h_{1}\right\},\left\{h_{1}, h_{3}\right\}\right], \\
& H\left(e_{3}\right)=\left[\left\{h_{3}, h_{4}\right\},\left\{h_{3}, h_{4}\right\}\right], \\
& H\left(e_{4}\right)=\left[\left\{h_{5}\right\},\left\{h_{1}, h_{3}, h_{5}\right\}\right], \\
& H\left(e_{5}\right)=\left[\left\{h_{4}\right\},\left\{h_{1}, h_{4}, h_{6}\right\}\right], \\
& H\left(e_{6}\right)=[\varnothing, \varnothing], \\
& H\left(e_{7}\right)=[U, U] .
\end{aligned}
$$

Then $\left(H, E_{2}\right)$ is an interval soft set over $U$. Compared with interval soft set $\left(G, E_{1}\right)$ in Example 5.10, we know that $\left(H, E_{2}\right) \approx_{2}\left(G, E_{1}\right)$. Since $G\left(e_{7}\right)=$ $[U, U]$, this means that all of houses under consideration are modern, therefore, $e_{7}$ is not considered in the decision problem to select houses, we can apply the above general decision method by using soft set $\left(H, E_{2}\right)$ (from Step 1 to Step 3).

\section{Summary}

Interval sets provide a new means for representing partially known concepts or for approximating undefinable concepts or complex concepts. The soft set theory is also a new mathematical tool for dealing with uncertainties that is free form the difficulties that have troubled the usual theoretical approaches. In order to better play the role of these two mathematical tools, we proposed a new concept of in- 
terval soft set. The paper illustrate the tabular representation of interval soft sets, apply the interval soft sets to solve a decision making problem, and show that interval soft sets have more flexibility in the expression of uncertainty compared to soft sets. As a basic theoretical research of interval soft sets, we investigate some operations of interval soft sets and construct some lattice structures. Moreover, we introduce the notion of soft equality (I-type and IItype)in interval soft set theory and establish quotient algebra by soft equality relation. Finally, by some examples, we discuss the preconditioning method of decision making by using soft equality relations. In future, we will study the interval soft sets combining with fuzzy sets, rough sets and (intuitionistic) fuzzy rough sets $\left(\operatorname{see}^{28}\right)$.

\section{Acknowledgments}

This work is supported by National Natural Science Foundation of China (No. 61175044), Science and Technology Commission of Shanghai Municipality (STCSM, No. 12510501700) and Science and Technology Program of Shanghai Maritime University.

\section{References}

1. M. I. Ali, F. Feng, X. Liu, W. K. Min, M. Shabir, "On some new operations in soft set theory," Computer$s$ and Mathematics with Applications, 57, 1547-1553 (2009).

2. D. G. Chen, E. C. C. Tsang, D. S. Yeung, X. Z. Wang, "The parameterization reduction of soft sets and its applications," Computers and Mathematics with Applications, 49, 757-763 (2005).

3. F. Feng, Y. B. Jun, X. Z. Zhao, "Soft semirings," Computers and Mathematics with Applications,56, 26212628 (2008).

4. F. Feng, C. X. Li, B. Davvaz, M. I. Ali, "Soft sets combined with fuzzy sets and rough sets: a tentative approach," Soft Computing, 14, 899-911 (2010).

5. Y. B. Jun, "Soft BCK/BCI-algebras," Computers and Mathematics with Applications, 56, 1408-1413 (2008).

6. Y. B. Jun, C. H. Park, "Applications of soft sets in ideal theory of BCK/BCI-algebras," Information Sciences, 178, 2466-2475 (2008).

7. P. K. Maji, R. Biswas, A. R. Roy, "Fuzzy soft sets," The Journal of Fuzzy Mathematics, 9, 589-602 (2001).
8. P. K. Maji, R. Biswas, A. R. Roy, "Soft set theory," Computers and Mathematics with Applications, 45, 555-562 (2003).

9. P. K. Maji, A. R. Roy, R. Biswas, "An application of soft sets in a decision making problem," Computer$s$ and Mathematics with Applications, 44, 1077-1083 (2002).

10. D. Molodtsov, "Soft set theory-First results," Computers and Mathematics with Applications, 37, 19-31 (1999).

11. R. E. Moore, "Interval Analysis," Prentice-Hall, Englewood Cliffs, New Jersey, 1966.

12. Z. Pawlak, "Rough sets," International Journal of Computer and Information Science, 11, 341-356 (1982).

13. Z. Pawlak, "Rough sets: Theoretical Aspects of Reasoning About Data," Kluwer Academic Publishers, Boston, 1991.

14. Z. Pawlak, A. Skowron, "Rudiments of rough sets," Information Sciences, 177, 3-27 (2007).

15. D. W. Pei, D. Q. Miao, "From soft sets to information systems," Proceedings of the IEEE International Conference on Granular Computing, 2, 617-621 (2005).

16. K. Y. Qin, Z. Y. Hong, "On soft equality," Journal of Computational and Applied Mathematics, 234, 13471355 (2010).

17. A. R. Roy, P. K. Maji, "A fuzzy soft set theoretic approach to decision making problems," Journal of Computational and Applied Mathematics, 203, 412418 (2007).

18. A. Sezgin, A. O. Atagun, "On operations of soft sets,Computers and Mathematics with Applications," 61, 1457-1467 (2011).

19. X. B. Yang, T. Y. Lin, J. Y. Yang, Y. Li, D. J. Yu, "Combination of interval-valued fuzzy set and soft set," Computers and Mathematics with Applications, 58, 521-527 (2009).

20. Y. Y. Yao, "Interval-set Algebra for Qualitative Knowledge Representation," Proceedings of the 5th International Conference on Computing and Information, 370-375 (1993).

21. Y. Y. Yao and X. Li, "Comparison of Rough-set and Interval-set Models for Uncertain Reasoning," Fundamenta Informaticae, 27, 289-298 (1996).

22. Y. Y. Yao and S. K. M. Wong, "Interval Approaches for Uncertain Reasoning," Proceedings of the 10th International Symposium on Methodologies of Intelligent Systems, 381-390 (1997).

23. Y. Y. Yao and J. Wang, "Interval based Uncertain Reasoning using Fuzzy and Rough Sets," Advances in Machine Intelligence and Soft-Computing, 196-215 (1997).

24. Y. Y. Yao, "Interval sets and interval-set algebras," Proceedings of the 8th IEEE International Conference on Cognitive Informatics, 307-314 (2009). 
25. X. H. Zhang, X. Y. Jia, "Lattice-valued interval sets and t-representable interval set t-norms," Proceedings of IEEE International Conference on Cognitive Informatics, 333-337 (2009).

26. X. H. Zhang, "Fuzzy Logics and Algebraic Analysis," Science Press, Beijing, 2008.

27. X. H. Zhang, Q. Fu, "Interval soft sets," Proceedings of 2nd IEEE International Conference on Cloud Com- puting and Intelligence Systems, 74-78 (2012).

28. X. H. Zhang, B. Zhou, P. Li, "A general frame for intuitionistic fuzzy rough sets," Information Sciences, 216, 34-49 (2012).

29. P. Zhu, Q. Y. Wen, "Probabilistic soft sets," Proceedings of 2010 IEEE International Conference on Granular Computing, 635-638 (2010). 